\title{
El preventorio infantil de montaña de la Cruz Roja Chilena
}

\author{
Autor: EDUARDO OSTORNOL ${ }^{1}$ \\ Comentario: LUISA SCHONHAUT B. ${ }^{2}$ \\ 1. Vice-Presidente del Comité Central de la Cruz Roja Chilena. \\ 2. Profesor Asistente, Magíster Salud Pública, Departamento de Pediatría Norte, Facultad de Medicina, Universidad de Chile.
} Palabras clave: Tuberculosis, Preventorios,
historia pediatría .

Key words: Tuberculosis, pediatric history.

\section{Introducción}

El 15 de diciembre de 1929, bajo iniciativa de la Cruz Roja Chilena y siguiendo estrictamente las recomendaciones internacionales, abrió sus puertas el Preventorio Infantil de Montaña en San José de Maipo, para el cobijo y atención de niños de escasos recursos que fuesen contacto de tuberculosis, pero sin evidencias de infección activa.

"Creado bajo la iniciativa del doctor E. Ostornol y dirigido por el mismo con admirable dedicación y cariño por la obra, sus beneficios han sido bastante apreciables. No hace mucho tiempo hemos tenido ocasión de visitarlo y hemos podido imponernos de los grandes progresos materiales realizados desde su instalación. El pabellón principal recientemente inaugurado ha sido muy bien concebido, y reúne todas las condiciones que exige su objeto, siendo además de muy hermoso aspecto." (Osorio 1933) ${ }^{1}$

Tras seis décadas de inagotable funcionamiento, debido a la falta de recursos para su mantención, en el año 1992, el edificio debió cerrar sus puertas ${ }^{2}$, siendo declarado Monumento Histórico Nacional el año 2004.

\section{El preventorio infantil de montaña de la Cruz Roja Chilena}

EDUARDO OSTORNOL

Vice-Presidente del Comité Central

de la Cruz Roja Chilena

En San José de Maipo, en plena montaña, a dos kilómetros de la Villa, en el camino al Volcán y a unos trescientos metros de la Casa de Salud, la Cruz Roja Chilena ha establecido y mantiene abierto, desde el 15 de diciembre del año pasado un Preventorio Infantil, destinado a preservar a los niños del avance de la tuberculosis, casi siempre mortal, para estos organismos débiles y abandonados, a sus propios y escasos recursos.

Con visión clara y con pleno concepto de la obra que el país necesita, con mayor urgencia, (si quiere salvar el futuro de la población) la Cruz Roja Chilena ha querido iniciar, con la fundación de este Preventorio, la campaña de preservación de la infancia en contra de la Tuberculosis. Es el primer Preventorio que se establece en esta Montaña que se le dio al país, 
como baluarte y defensa de la salud de sus habitantes y que nosotros, no hemos sabido ni querido aprovechar.

No será, tampoco, el único que se funde en el país, porque la Cruz Roja Chilena esta dispuesta a encarar de frente este grave problema de salud para el niño chileno, multiplicando estos Preventorios, a lo largo del territorio y en todas aquellas regiones, que son muchas, en las cuales se reúnan las condiciones de clima, de temperatura, de vida y de fácil acceso que sean favorables a la instalación de estos servicios y al mantenimiento de su función.

Este Preventorio fue construido, en el seno de un bosque frondoso, formado por la mano del hombre, a quien la naturaleza y el clima de esa región le prestó el auxilio de sus dones maravillosos: Todo en ese Preventorio ofrece salud y vida; el aire es puro y fresco; el sol es radiante y la atmósfera se presenta siempre clara y transparente. La existencia del niño se desenvuelve en medio de todas las comodidades que ha sido posible proporcionarle para que su permanencia allí sea grata y saludable.

El niño vive, todo el día, en plena naturaleza, juega, corre y se alimenta al aire libre; tiene una esplendida y completa cancha y plaza de juegos y goza de un reposo, bien distribuido y reglamentado que le permite aprovechar bien su alimentación y favorece su nutrición.

El objetivo que se persigue, en todo momento y en cada acción, es robustecer al niño, modificar su constitución, restaurar sus energías: Cada acto que el niño ejecuta, cada movimiento que desarrolle, debe estar dirigido a conseguir este fin.

La alimentación es abundante, bien condimentada y sana y está calculada para restablecer y engordar al niño, en el menor tiempo posible.

Los servicios higiénicos, los dormitorios, el baño, los ejercicios, han sido estudiados y establecidos, en relación, con las necesidades del niño y con el buen deseo de formar hábitos nuevos en estos niños que, no conocen las practicas del aseo diario, que desconocen los beneficios del baño y de la limpieza y que deben aprender nuevas costumbres para el uso de ellos mismos y para ejemplo y enseñanza en los hogares, a los cuales volverán después, llenos de salud y con hábitos de higiene y aseo personal.

Los niños que se admiten en este Preventorio, son seleccionados en las Escuelas Públicas, por las enfermeras visitadoras de la Cruz Roja de las Mujeres de Chile y son examinados, después de una minuciosa encuesta, por Médicos del Servicio. de la Cruz Roja.

A cada uno de estos niños se les somete a un examen clínico y radiológico y se les anota, en una hoja especial, el estado de sus ganglios y de su parénquima pulmonar.

Es bien entendido que, tratándose de un Preventorio, no se admite a ningún niño que se encuentre ya tuberculoso y con lesiones ya contagiosas. Tampoco, se admite ni se recibe a ningún niño que presente síntomas o manifestaciones de alguna otra enfermedad de la piel o de otros órganos, que puedan ser fácilmente trasmitidos a los otros niños.

Para la admisión de los niños que pueden ingresar al Preventorio, su Dirección ha fijado condiciones que forman parte del Reglamento Interno de estos Servicios.

Las condiciones de vida que llevan estos niños en el Preventorio, deben haber sido bien estudiadas y deben haber sido mejor cumplidas, cuando desde el día en que abrió sus puertas este Preventorio (15 XII 29) hasta esta fecha y habiendo pasado por el en este tiempo, tres colonias de sesenta a setenta niños cada una, no ha habido que lamentar ningún accidente de importancia, ni cuidar a ningún enfermo febril.

En este Preventorio, no se ha dejado nada que no haya sido previsto y estudiado. Los niños gozan de toda clase de libertad, dentro de la disciplina y del respeto que imponen las prácticas del servicio.

Las horas reglamentarias de distribución de los alimentos, del reposo, del baño, como las horas de levantarse y de acostarse, han sido estudiadas y aplicadas en relación con la edad del niño y las condiciones climatéricas de la región.

El Establecimiento lleva un Libro de ingreso en el cual se anotan los principales antecedentes de cada niño al llegar, y se deja constancia, también de las observaciones que la vida del niño ha merecido, durante su permanencia en el Preventorio. 
A su llegada al Preventorio, el niño es introducido al Pabellón de Recepción y de Limpieza; en el cual se le toma el peso y la talla, se hacen las anotaciones establecidas en el Libro de ingreso; se le baña y se le cambia, toda clase de ropa, vistiendo la ropa del Establecimiento que es uniforme para todos.

La ropa del niño, se desinfecta y se guarda numerada, con el número de orden correspondiente al nuevo pupilo.

Desde ese momento, el niño pertenece al Establecimiento, pero, no entra de lleno, a la vida de los otros niños. A pesar de los exámenes médicos y de los certificados con que llega al Preventorio, este niño debe quedar en observación, durmiendo separado del dormitorio común y sujeto a la minuciosa observación de la Dirección.

La edad del niño debe fluctuar, entre seis y diez años, para ser admitido: No se ha podido ensanchar más el límite de edad, por razones de índole interna y practica: Los niños menores de seis años, no saben vestirse ni manejarse solos; habría entonces, necesidad de aumentar el número y la calidad del personal; por otra parte, los niños mayores de diez años son difíciles, irreductibles de carácter para el Personal directivo que es femenino y además, tienen el peligro de conocer mucho la vida, son muy precoces y suelen llevar y tener vicios y hábitos perniciosos para los otros niños.

Todo niño al llegar al Preventorio, debe ir premunido con una tarjeta de introducción, entregada por el Comité Central de la Cruz Roja Chilena, después de los exámenes a que ha sido sometido y que demuestren:

1) Que tiene necesidad de ingresar al Preventorio, por salud; 2) Que carece de recursos; y 3) Que no padece de ninguna enfermedad infecciosa que pueda ser perjudicial para los otros niños.

Se ha querido, de este modo, evitar la sorpresa de una enfermedad transmisible, tan frecuente en los niños, que pudo estar enmascarada, en el memento del examen y aparecer después, a su ingreso al Preventorio.

Iguales precauciones se toman con las personas de la familia de los niños que tienen autorización para visitarlos, en los días domingos o festivos, impidiendo la entrada de los niños que tienen tos u otros síntomas sospechosos.

La vida interna se desarrolla, diariamente dentro de los principios educativos de higiene, de limpieza, de cultura física y de enseñanza cívica y moral que debe contribuir a formar los nuevos hábitos con que estos niños deben regresar a su hogar: De este modo, la permanencia de un niño en el Preventorio no le significa, sólo, mejoramiento en las condiciones de salud, sino que también, enseñanzas de provecho para su vida futura, porque les forma una nueva personalidad, con nociones y practicas saludables de aseo personal, de orden y disciplina en el servicio diario y de cultura moral y cívica. Los niños son examinados, en muchas ocasiones, muchos días antes de su ingreso al Preventorio en sus relaciones con los otros niños y con las personas que dirigen el Preventorio.

De este modo, el Preventorio entra a ser una Escuela que enseña, educa y difunde conocimientos útiles y necesarios para la vida del niño y para los hogares, de los cuales vienen y que, en general, son refractarios a estas costumbres o no las cultivan.

Los niños se levantan, alrededor de las 7:30 horas de la mañana en el invierno y después de lavarse los dientes, proceden a su lavado con agua fría y jabón. En el invierno, este lavado deberá hacerse con agua temperada, porque el agua es muy fría y lastima la piel.

Después de vestidos, los niños, bajo la vigilancia de la Inspectora General proceden a deshacer sus camas para ventilarlas, mientras ellos salen a tomar su desayuno y a practicar ejercicios de respiración y de gimnástica.

A las 9 de la mañana, los niños entran al dormitorio, que ha sido ventilado y que, desde esa hora hasta el momento de acostarse permanece abierto, para hacer sus camas, bajo la vigilancia de la Inspectora y con la ayuda del personal en servicio, que se ocupa del aseo y limpieza del piso, de los baños y lavatorio.

Como se ve, los niños no hacen servicios de carácter general en el Preventorio, pero se les ha querido acostumbrar y que ellos mismos se sirvan para enseñarlos a vivir y desempeñarse solos en su vida futura.

Desde las 9:30 hasta las 11:30 hora del almuerzo, los niños viven al aire libre, en juegos, 


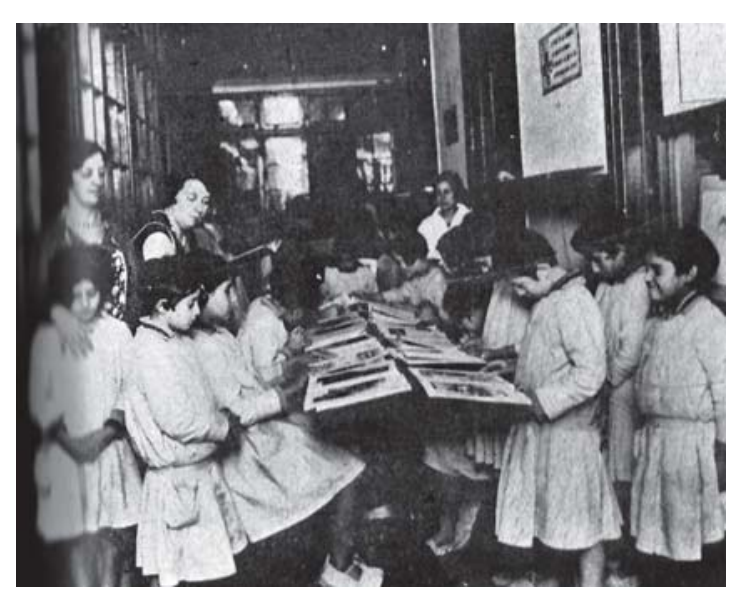

Figura 1. Niños en la sala de lectura.

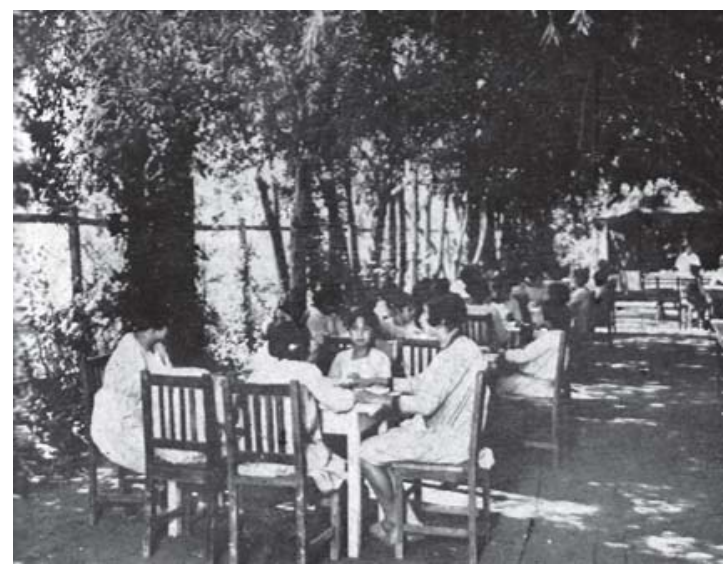

Figura 3. Almuerzo en el parque.

ejercicios, carreras y saltos. Al sonido de la campana que anuncia la hora del almuerzo, todos los niños corren a lavarse las manos y a peinarse, a fin de presentarse limpios y correctos a la mesa.

El almuerzo es servido al aire libre, bajo los árboles y en mesas, calculadas para seis niños. En los cajones de estas mesas, que están cubiertas con hule blanco, los niños encuentran sus cubiertos y su servilleta, guardados en un servilletero de género, con el número correspondiente a su asiento y que lleva el número de orden de cada niño.

El Servicio de platos se hace en cada mesa por uno de los niños que se va cambiando por

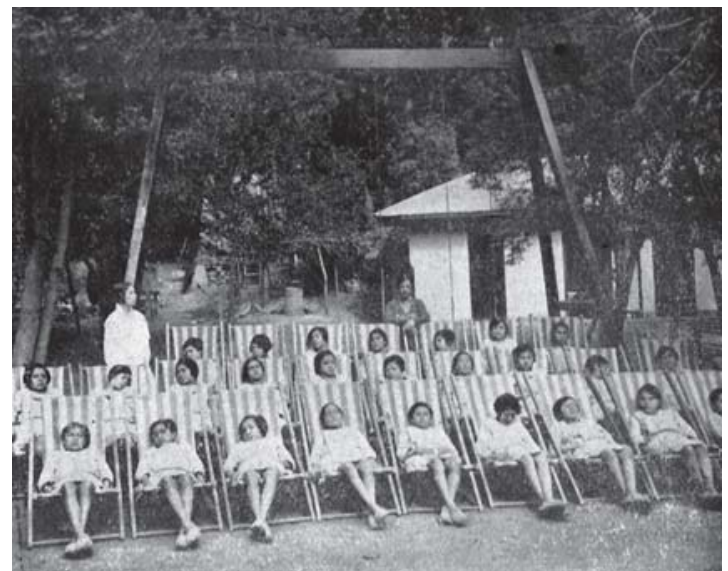

Figura 2. Niños en reposo.

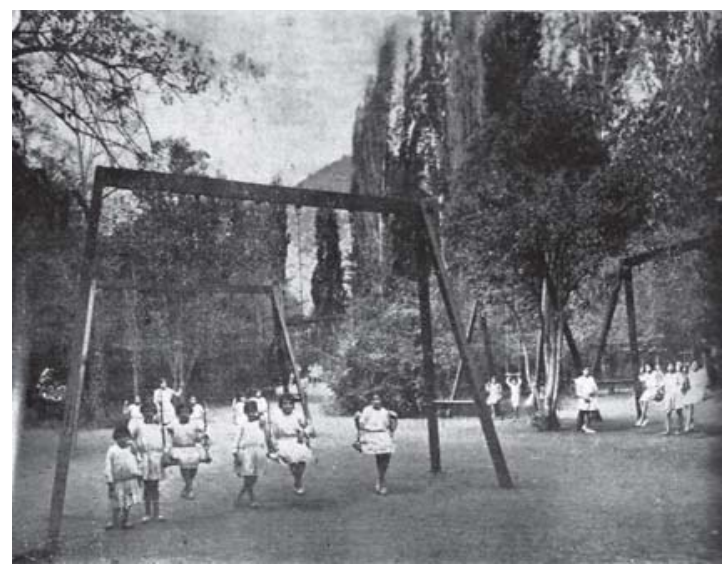

Figura 4. Juegos infantiles en el parque.

turno semanal. Terminado el almuerzo, durante el cual los niños tienen libertad para hablar y conversar, sin desorden y en el cual reciben instrucciones y enseñanzas sobre la forma como deben comer y mantenerse en la mesa, cada niño debe limpiar sus cubiertos, con agua caliente que se lleva, especialmente, a la mesa y guardarlos con su servilleta en su servilletero, respectivo.

Después del almuerzo, el reposo de media hora es obligatorio: Cada niño tiene su silla de reposo con su número, a fin de establecer la responsabilidad necesaria en caso de destrucción o falta de cuidados.

La tarde se dedica, después del reposo, a 
juegos, lecturas y paseos al aire libre, hasta la hora del te que se sirve a las 16 horas y que puede ser cocoa, ulpo caliente, café o te con leche, pan, galletas y frutas.

La comida se sirve a las 18 hrs 30 y después de comer, los niños se entretienen, siempre, al aire libre hasta las 20 hrs 30 ó 21 hrs, en que se dirigen al dormitorio para acostarse.

El lavado de los dientes es de obligación, antes de acostarse, para lo cual cada niño encuentra, al alcance de su mano, en la consola que tiene al lado de su cama, un vaso, un cepillo para dientes y una caja de polvos. Tiene, además, fuera de esto, una jabonera con jabón, una peineta y una toalla, útiles todos de carácter individual y de uso personal para cada niño.

Durante la noche, los niños duermen, con las ventanas abiertas, con una pequeña luz de emergencia y bajo la inmediata vigilancia de la Inspectora General que duerme en una pieza vecina al dormitorio y con la llave de los focos eléctricos al alcance de su mano para alumbrar, en casos de accidentes o pánicos nocturnos y con un teléfono directo para comunicarse con la Dirección, en caso de enfermedad, de accidentes o de peligro.

Los niños se encuentran completamente cuidados y garantidos en el Preventorio, tienen allí al alcance de sus manos, los juegos que ellos anhelan, están limpios, bien alimentados, sin trabajo ni ocupación ninguna y dedicados, exclusivamente a reponerse, a reconstituirse: este es el objeto de su ingreso al Preventorio y debe ser el único fin de su vida diaria.

Así lo ha estimado la Dirección del Preventorio que no escatima cuidado, alimentación, ejercicio ni reconstituyentes de carácter alimenticio o medicamentoso para alcanzar el aumento de peso de los niños y el mejoramiento del estado general de su salud.

En esta forma, ha comprendido la Cruz Roja Chilena su papel de defensa en favor del niño y en contra de la Tuberculosis y confía en que, manteniendo durante todo el año abierto y en servicio el Preventorio de San José de Maipo, creando y habilitando otros semejantes en otras regiones del país, ya sea en la montaña, en el campo o en el mar, se ha de conseguir arrebatar a la peste blanca, su cosecha anual de niños que tanto compromete el futuro de Chile, su crecimiento y su progreso.

\section{Comentario}

La tuberculosis, conocida en tiempos de Hipócrates como "tisis", consunción y posteriormente como "peste blanca", ha cobrado más victimas que cualquier otra enfermedad a lo largo de la historia de la humanidad; de hecho, hace tan sólo 70 años, era responsable de la cuarta parte de las muertes notificadas en nuestro país ${ }^{3}$.

No obstante la antigüedad de la tuberculosis, los avances en su etiología, control y tratamiento son relativamente nuevos. Previo al descubrimiento de Koch el año 1882 y mucho antes de la introducción de la estreptomicina en 1944, existía una percepción de la transmisibilidad de la enfermedad y de poca utilidad de los tratamientos medicamentosos.

A fines del siglo XIX se crearon los Sanatrios Antitubercuosos, donde se administraba una terapia orientada a detener la consunción mediante el reposo, aire libre y adecuada alimentación en la "Cura Sanatorial"; así se cumplía simultáneamente el objetivo de aislar al enfermo del resto de la población ${ }^{4}$.

"La terapéutica medicamentosa no juega un papel importante en la tuberculosis infantil. Pocas enfermedades habrán conocido, como la tuberculosis un mayor número de agentes curativos. Pero tantos entusiasmos y esperanzas como en ellos se pusieron, no se han visto compensados: al contrario, pronto ha venido el descrédito. Pero es difícil conformarse con una actuación pasiva.

... Tiene en general una influencia sugestiva que tratándose de una enfermedad crónica, es digna de atención pues ayuda al proceso curativo. Su acción se dirige especialmente a levantar el estado general -medicación tónica, roborante- a mejorar el apetito y a calmar molestias de naturaleza catarral" (Osorio 1934)

Como medida profiláctica, para aquellos niños que habían tenido contacto con el bacilo pero sin desarrollo de la enfermedad activa, se diseñaron los Preventorios Infantiles. Según re- 
lata Osorio ${ }^{1}$, estos establecimientos tuvieron su origen a comienzos del siglo XIX en las "escuelas al aire libre", organismos que se extendieron a lo largo de todo el mundo y cuya finalidad era mejorar las condiciones generales del niño, pero desde un enfoque más bien pedagógico:

"Las escuelas al aire libre fueron creadas para mejorar las condiciones del niño débil, desnutrido, hipotrófico; para el convaleciente de enfermedades infecciosas, anémico o fatigado por el trabajo escolar, realizado muchas veces en locales antihigiénicos. Pero hoy día, teniendo muy presente todos estos factores, el propósito fundamental que se persigue es la lucha contra la tuberculosis. En la selección de los niños que a ellas deben concurrir, además del estado nutritivo, se tiene especialmente en cuenta el pertenecer a familia de tuberculosos o presentar reacción positiva a la tuberculina. Estas condiciones se extreman tratándose de las escuelas de tipo internado; por eso se llaman hoy día Preventorios antituberculosos y están comprendidos entre los órganos de la lucha antituberculosa.

Son, como su nombre lo indica, organismos de profilaxis y no se admiten en ellos niños enfermos con lesiones pulmonares evolutivas, contagiosas. El preventorio trata de alejar al niño indemne aún o ya infectado, del foco de contagio familiar y sustraerle a las condiciones desfavorables del medio, para evitar la superinfección.

Lo que se persigue es no sólo obtener un aumento ponderal, sino una elevación del Standard de salud, comprendido el desarrollo físico y muscular armonioso, la corrección de los defectos del hábito exterior, el entrenamiento contra la fatiga, el equilibrio entre las funciones orgánicas, la mejor defensa o relativa inmunidad contra las infecciones, especialmente de las vías respiratorias; y por último, en la esfera psíquica, se trata de hacer renacer la viveza, la animación, la alegría propia del niño.

Todo esto no se puede conseguir con el empleo unilateral sistemático de uno u otro de los medios de estimulación, ya sea la helioterapia, los baños o los ejercicios físicos. Tal terapéutica seria necesariamente parcial y no daría sino mejorías pasajeras incompletas. No basta sólo el aire puro en el pulmón sino que hay que recurrir además al estímulo de la piel en toda su superficie. Es por eso que la acción del preventorio se funda en el empleo paralelo o alternado de los diferentes agentes físicos naturales; sol, aire, agua, movimiento. La terapéutica medicamentosa es absolutamente secundaria.

Los elementos esenciales que informan la higiene y la cura preventorial, son los siguientes:

1) Clima; marítimo, de campo o llanura, de montaña.

2) Aireación permanente: vida al aire libre.

3) Helioterapia: cura de sol sobre el cuerpo desnudo.

4) Ejercicios físicos: gimnasia, juegos, canto.

5) Hidroterapia: baños, duchas o abluciones frías.

6) Alimentación: racional, abundante, a horas regulares.

7) Cura de reposo: en decúbito horizontal, siesta, al aire libre.

A esto se agrega la labor educativa, pedagógica que consiste en clases o charlas instructivas, cortas, hechas al aire libre. Enseñanza cívica, moral, y de higiene personal. Corrección de modales y malos hábitos. Pequeños trabajos (jardinería, por ej), y labores de economía domestica, (niñas).

... Para terminar, quisiéramos reproducir las palabras con que Armand-Delille, gran animador de la obra de las escuelas al aire libre y los preventorios, en Francia, resume los resultados obtenidos con el plan que acabamos de bosquejar. Ellas concuerdan absolutamente con las publicadas por todos los que se han ocupado de esta misma cuestión.

$\mathrm{Al}$ ingreso se presenta un niño de tejidos blandos, con sus músculos flácidos apenas desarrollados, con el dorso gibado, los omoplatos salientes, con el vientre abultado, caído en su parte inferior por atonía de la pared. La expresión de la cara es triste, fatigada, los rasgos extenuados, caídas las comisuras de los labios. Después de tres o cuatro meses de cura nos encontramos ya con un niño de tejidos firmes; sus músculos se sienten tensos bajo una piel tersa y bronceada; su columna se ha enderezado y de pie ya no presenta ni cifosis ni lordosis; la cabeza se mantiene bien erecta sobre los hombros; el vientre se ha aplanado. En fin, la 
fisonomía revela brío, animación, alegría; la cara esta llena, la expresión es sonriente.

La moral del niño ha sido igualmente transformada: callado, triste a su llegada, a menudo taimado, reconcentrado, o bien difícil, insubordinado, se despeja con la libertad, el contacto con la naturaleza y la influencia de los ejercicios gimnásticos en común. El muchacho adquiere valor físico y moral. Se muestra contento, activo, franco, complaciente, siempre dispuesto a prodigar algún servicio a sus maestros o a sus camaradas.

En algunos meses se observa no solamente cómo aumenta el peso y cómo se broncea la piel, sino también, y este es el hecho más notable, cómo se opera una transformación en el hábito y la expresión" (Osorio Preventorios).

\section{Referencias}

1.- Osorio A: Sobre el programa de acción de los preventorios antituberculosos. Rev Chil Pediatr 1933; (7).

2.- Von Bennewitz R: Cruz Roja en Chile. Preventorio de San José de Maipo [accedido 15 diciembre 2007] disponible en http://www.dedaldeoro.cl/ed40-cruz_roja.htm

3.- Vargas $N$ : Historia de la pediatría chilena: crónica de una alegría. Editorial Universitaria. Santiago de Chile 2002.

4.- Farga V: La conquista de la tuberculosis. Rev Chil Enferm Respir 2004; 20 (2): 101-8. http://www.scielo. cl/scielo.php?script=sci_arttext\&pid=S07177348200 4000200009\&lng=es\&nrm=iso

5.- Osorio A: Tratamiento de la tuberculosis en el niño. Rev Chil Pediatr 1934; 2 (9). 